\title{
Representación Infantil de la Ciencia usando el Test Dibujando un Científico (DAST). Posibilidades de Cambios desde la Comunicación Científica
}

\author{
Lorena B. Valderrama ${ }^{(1)}$, Teresa P. Vernal-Vilicic ${ }^{(2)}$ y Leyla Méndez-Caro ${ }^{(3)}$ \\ (1) Programa de Formación General, Universidad Diego Portales, Av. Manuel Rodríguez Sur 253, Santiago, \\ Chile. (e-mail: lolo.valderrama@gmail.com) \\ (2) Universidad Finis Terrae, Avenida Pedro de Valdivia 1509, Providencia, Santiago, Chile. \\ (e-mail: tvernalv@uft.edu) \\ (3) Departamento de Ciencias Sociales, Artes y Humanidades, Campus Coloso Universidad de Antofagasta \\ 02800, Antofagasta, Chile. (e-mail: leyla.mendez@uantof.cl)
}

Recibido Ago. 13, 2016; Aceptado Sep. 27, 2016; Versión final Oct. 18, 2016, Publicado Dic. 2016

\begin{abstract}
Resumen
Este artículo presenta los resultados de una intervención para analizar y proponer modificaciones sobre la representación infantil de la ciencia. La intervención se realizó como parte de una actividad de educación no formal del programa Desarrollando y Liderando Talentos Académicos, DeLTA, de la Universidad Católica del Norte en Chile. Para evaluar la representación infantil de la ciencia se utilizó el Test Dibujando un Científico (DAST, Draw A Scientist Test), usada a nivel internacional para medir estereotipos de la ciencia en población infanto-juvenil. La evaluación de la intervención permitió constatar la efectividad de la metodología de medición de estereotipos y facilitó la propuesta de modificaciones sobre la representación infantil de la práctica científica. Finalmente, en el artículo se propone la enseñanza de la comunicación pública de la ciencia en la población infantil como una forma de intervención profunda que posibilita cambios positivos de estereotipos y de representaciones sociales sobre prácticas del quehacer científico.
\end{abstract}

\section{Child Representation of Science using the Draw-a-Scientist Test (DAST). Possibilities of Changes from Scientific Communication}

\begin{abstract}
This article presents the results of an intervention to analyze and propose modifications about child representation of science. The intervention was carried out as part of an activity of the non-formal education program Developing and Leading Academic Talent, DeLTA of the Universidad Católica del Norte in Chile. To assess children's representation of science the methodology known as Draw a Scientist Test (DAST) internationally used to measure stereotypes of science in child population was employed in this study. The evaluation of the intervention allowed confirming the effectiveness of the methodology for measuring stereotypes and helped in proposing modifications about child representation on scientific practice. Finally, the article proposed that the teaching of public communication of science among children be a form of profound intervention that facilitates positive changes of stereotypes and of social representation of scientific work.
\end{abstract}




\section{INTRODUCCIÓN}

La manera en que representamos el mundo se ha convertido en un foco de investigación importante dentro de las ciencias sociales y humanas, consolidándose en los últimos 55 años (Oliveira, 2009). Las representaciones sociales (en adelante RS) son entendidas como un corpus organizado de conocimientos que hacen inteligible la realidad física y social en el entramado de las relaciones cotidianas bajo la triada Individuo (Ego) - Otro (Alter) - Objeto, (Moscovici, 2005) siendo Moscovici, uno de sus principales exponentes quien realizó un giro epistemológico en el estudio de éstas, distanciándolas del estudio tradicional de actitudes en tanto concepto estático y poco social (Wagner y Flores-Palacios, 2010) para resituarlo en términOs socioculturales sobre la base de normas y valores grupales (Cortassa, 2010). En efecto, las representaciones sociales serán entendidas como un saber o conocimiento de sentido común socialmente elaborado o compartido en el que se articulan las esferas subjetiva, intersubjetiva y transubjetiva (Jodelet, 2011).

Las RS poseen una capacidad de transformación y deben, por lo tanto, ser entendidas como fenómenos dinámicos, que bajo condiciones sociales determinadas, propiciarían la transformación en el pensamiento, toda vez que éste es entendido como un fenómeno cultural e históricamente construido a través de la comunicación, las tensiones y las transformaciones sociales (Marková, 2006). En el caso de la población infantil, niños y niñas interiorizan el mundo en que viven y, a través de esto, pueden conocer parte de él, es decir construyen representaciones sociales (Lay y Montañes, 2013). Las RS propician la naturalización de ciertas imágenes y categorías sociales que delimitarían desde temprana edad los campos de acción de los/as niños/as, en tanto sesgo interviniente de las posibles interacciones y escenarios de vinculación, repercutiendo así en sus identidades sociales (Pizzo, Slobinsky y Panzera, 2006). Este proceso, no está exento de las relaciones de poder y las RS son legitimadas a través de instituciones como la familia y la escuela (Jodelet, 2011) entidades claves en los procesos de socialización primaria y secundaria en la que se producen los primeros procesos de interacción y producción simbólica.

Los medios de comunicación juegan un papel crucial en la reproducción de las RS, ya que "buscan reafirmar un modelo mental específico que contiene la representación social de un objeto, suceso o persona" (Mayorga y León, 2007, p. 117). Se constituyen como transmisores de ideologías dominantes y facilitadores de la producción de cogniciones sociales (Van Dijk, 2009) y estabilizan ciertos sentidos sociales sobre el mundo y su funcionamiento desde de sus aspectos interindividuales, institucionales y de masas (Domínguez-Gutiérrez, 2006).

\section{Representaciones sociales de la ciencia}

En el marco de la relación entre las representaciones sociales de la ciencia, algunos estudios han revelado que las estrategias de comunicación de la ciencia legitiman un tipo de conocimiento, favoreciendo ciertas áreas del saber (Domínguez-Gutiérrez, 2006; Manzini, 2003). Otros estudios han determinado una alta importancia de la labor de la comunicación y la educación de las ciencias en la configuración de determinadas representaciones sociales de la ciencia y de una alta presencia de estereotipos sobre el quehacer científico en la población infantil (Schibeci, 1986; Ruiz-Mallén y Escalas, 2012; Vernal y Valderrama 2014; Pujalte et. al 2014).

Un ejemplo de lo anterior se observa en los reportes de Tsai (2002) y Pujalte et.al, (2014) quienes señalan que las representaciones inadecuadas de profesoras/es sobre la ciencia y su enseñanza imponen ciertos efectos sobre las representaciones de las/os estudiantes, siendo el cambio de éstas un prerrequisito para lograr el cambio de concepciones acerca de la naturaleza de la ciencia. Asimismo, un estudio sobre la imagen de científicas/os a nivel secundario, dio cuenta de imágenes altamente estereotipadas en que resalta la imagen de científicos varones "blancos", quienes trabajan solos dentro de un laboratorio, además de ser jóvenes y sonrientes (González, et.al, 2009).

Una RS interesante en este escenario es aquella edificada sobre ciencia y su segmentación por sexo, en la que se tiende a invisibilizar el quehacer de las mujeres en este ámbito, tal como se reporta en un estudio reciente sobre las representaciones de las mujeres científicas en la prensa española, donde se observa que los diarios analizados dedican únicamente el $2,6 \%$ de sus páginas a información sobre ciencia y que, dentro de éstas, las mujeres protagonizan el $14,3 \%$ de los artículos frente al $70,6 \%$, que se centra en los hombres (Aladro, et. al, 2014). De esta manera, Torres (2005) enfatiza en la importancia de considerar el contexto social e histórico así como la opinión pública como entes activos en la construcción de las representaciones sociales sobre la ciencia y tecnología, toda vez que las RS vehiculizarían la comprensión pública de la ciencia, así como permitirían explorar aquella brecha entre ciencia y sociedad. A través de éstas podemos "examinar mecanismos operativos mediante los cuales se generan las representaciones grupales y cómo éstas, a su vez, se articulan en las representaciones subjetivas, permeando los modos de percepción, los 
juicios y actitudes de los individuos que los integran" (Cortassa, 2010, p.41). En este sentido las RS, también nos permitirían una forma de apropiación social de la ciencia en diversos niveles y estadios.

Desde la década de 1950, se ha determinado que existe una imagen estereotípica de las personas dedicadas a las ciencias: un hombre de mediana o avanzada edad, con bata blanca y lentes, que trabajaba en un laboratorio realizando experimentos químicos (Mead y Metraux, 1957). Estudios con resultados similares han sido ejecutados entre la población infanto-juvenil durante las últimas cuatro décadas en países europeos o de habla inglesa, principalmente, como Estados Unidos, Australia, Inglaterra, España, República Checa, Grecia, Turquía, Portugal, Francia, Italia, Polonia, Rumania y Canadá (Schibeci y Sorensen, 1983; Schibeci y Riley, 1986; Fort y Varney, 1989; Mason et al., 1991; Sumrall, 1995; Neathery, 1997;Newton y Newton, 1998; She, 1998; Song y Kim, 1999; Andre et al., 1999; Barman, 1999; Joyce Farenga, 1999; Weinburg y Steele, 2000; Calabrese Barton, 2001; Morgan, Isaac y Sansone, 2001; Chavous, 2002; Finson, 2001, 2002; Fung, 2002; Mattern y Shau, 2002; Rubin, Bar y Cohen, 2003; Weinburg, 2003; Buldu, 2006; Christidou, 2006; Rodari, 2007; Chavous et al., 2008; Christidou, 2011; Christidou et al., 2012).

En el ámbito latinoamericano se han realizado en los últimos años estudios similares en varios países como Uruguay, Argentina, Bolivia, Colombia y Chile, entre otros (Polino, 2011; Pujalte et al., 2014; Bravo et al., 2014). Sin embargo, en el caso de Chile, los programas estatales de educación no formal para acompañar los procesos de valoración y divulgación científica (como Explora-Conicyt y Mil científicos, Mil aulas) no han realizado evaluaciones ni revisiones de su contribución en la construcción de estereotipos y representaciones de la ciencia. Entre estos estudios destacamos aquellos que se han concentrado en la medición de los estereotipos sobre la práctica científica presentes en la población infantil, como los que han sido evaluados por el Draw A Scientist Test (DAST), el cual se explicará en apartados posteriores. Esta prueba de dibujo ha sido desarrollada en la segunda mitad de la década de 1970 y perfeccionada en estudios posteriores (Chambers, 1983; Flick, 1990; Finson et al., 1995; Medina-Jerez et al., 2011; RuizMallén y Escalas, 2012; Vernal y Valderrama, 2014).

\section{Modificación de la imagen de la ciencia}

El intento por derribar estos estereotipos comenzó a desarrollarse a finales de la década de 1980, considerando la aplicación de dos pruebas DAST separadas por una intervención en la cual los participantes recibían la visita de un científico o científica que conversaba con ellos/as y les explicaba en qué consistía su trabajo. Resultados de estudios interventivos arrojaron que era posible modificar discretamente los estereotipos de género, puesto que si bien aumentó la presencia de mujeres científicas en los dibujos, este aumento no resultó significativo (Flick, 1990). Sin embargo otras intervenciones más profundas han mostrado cambios significativos en los estereotipos sobre las áreas disciplinares que componen el sistema ciencia. Tal es el caso del estereotipo sobre la ciencia como actividad de laboratorio que, tras intervenciones ad-hoc, permite cambios en la representación de la ciencia como una actividad que se puede, también, dar en terreno o al aire libre (Finson et al. 1995).

En este artículo proponemos la enseñanza de la comunicación pública de la ciencia en la población infantil como una forma de intervención profunda que posibilita cambios de estereotipos y representaciones sociales sobre prácticas del quehacer científico. El artículo contiene un detalle de la intervención realizada en población infantil durante el verano del año 2014, en el marco de un programa de educación no formal de la Universidad Católica del Norte. Dicha intervención ha sido evaluada por el DAST, usada a nivel internacional para medir estereotipos de la ciencia en población infanto-juvenil.

\section{METODOLOGÍA}

El presente estudio es de carácter cualitativo y consiste en el análisis de las posibilidades de cambio en cuanto a representaciones sociales de la ciencia en la población infantil, mediante una intervención de educación no formal centrada en el periodismo científico. En cuanto a las técnicas utilizadas para estudiar las representaciones sociales de la ciencia en la población infanto-juvenil, estas han variado entre cuestionarios, ensayo, dibujo y entrevistas, principalmente. Esta prueba consiste en solicitarles a niños y niñas que dibujen a una persona que haga ciencia, permitiendo con ello identificar estereotipos infantiles sobre el quehacer científico. Si bien este test de medición de la imagen de la ciencia no ha estado exento de críticas, por no quedar claro si mide los estereotipos presentes realmente en la población infanto-juvenil o bien sólo muestra imágenes construidas por los estudiados para que estos sean reconocibles por los solicitantes (Manzoli et al., 2006) el dibujo es una de las primeras destrezas del desarrollo infantil (Oaklander, 2008) y estudios previos han relevado el dibujo como una herramienta gráfica y proyectiva relevante para la expresión infantil para las edades entre 11 y 13 años (Vernal y Valderrama, 2014). 
Por esta razón la intervención fue evaluada mediante la prueba DAST realizada en dos etapas (preintervención y post-intervención). Todo el proceso se desarrolló siguiendo la pauta de la Ethical Guidelines For Educational Research de la British Educational Research Asociation (BERA, 2011) con el consentimiento informado y la previa autorización de los tutores correspondientes y asentimiento de los participantes. Al iniciar y al finalizar el curso de periodismo científico, a los/as estudiantes se les solicitó a los participantes que realizaran un dibujo bajo la consigna: "dibuja una persona que haga ciencia", sin hacer mención a distinciones de género y especificando que la dibujaran en un escenario que ellos/as consideraran habitual para este tipo de actividad.

Se construyeron categorías de análisis flexibles que fueron seleccionadas del último estudio internacional de este tipo (Ruiz-Mallén y Escalas, 2012). Estas categorías se distribuyeron en dos dimensiones: Agentes y Prácticas. La dimensión de los Agentes, corresponde a los estereotipos con respecto a quienes hacen ciencia e incluye categorías sobre el sexo de la persona que hace ciencia (Sexo), los rasgos y cualidades que configuran la manera de ser de la persona que hace ciencia (Personalidad) y aspecto exterior de la persona que hace ciencia (Apariencia), con sus respectivas subcategorías señaladas en la Tabla 1. La dimensión de las Prácticas corresponde a los estereotipos con respecto a el quehacer científico e incluye las categorías sobre la disciplina a la cual pertenece/ejerce la persona que hace ciencia (Área), las herramientas de trabajo que utiliza la persona que hace ciencia (Herramientas), el lugar de trabajo de la persona que hace ciencia (Locación) y actividad realiza la persona que hace ciencia (Tipo de actividad).

Tabla 1: Categorías creadas para las pruebas DAST 1 y 2

\begin{tabular}{|c|c|c|}
\hline & Categoría & Subcategoría \\
\hline \multirow{3}{*}{  } & Sexo & (hombre/mujer/indefinido). \\
\hline & Personalidad & (malvado/alocado/amigable/ triste/neutro/enojado). \\
\hline & Apariencia & $\begin{array}{l}\text { (Orden: desaliñado/ordenado; Rostro: vello facial/lentes/maquillaje; Vestimenta: bata } \\
\text { blanca/ropa casual/ropa técnica). }\end{array}$ \\
\hline \multirow{4}{*}{ 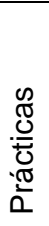 } & Área & $\begin{array}{l}\text { (química/biología/astronomía/física/matemática/geología/ } \\
\text { arqueología/cs.sociales/medicina/otras). }\end{array}$ \\
\hline & Herramientas & $\begin{array}{l}\text { (computadores/ microscopios/ telescopios/ lápices/ pizarras/ láser/ máscara/ tubos de } \\
\text { ensayo/ otros). }\end{array}$ \\
\hline & Locación & (oficina/ laboratorio/ observatorio/ aire libre). \\
\hline & Tipo de actividad & (experimentando/ pensando/ enseñando/ escribiendo/ exponiendo). \\
\hline
\end{tabular}

\section{Población objeto de Estudio}

Los participantes fueron 16 estudiantes de colegios públicos de Antofagasta (Chile), 8 niños y 8 niñas, con edades fluctuantes entre los 11 y 13 años, quienes provenían de colegios urbanos subvencionados de la región, quienes participaron del programa Desarrollando y Liderando Talentos Académicos, DeLTA, de la Universidad Católica del Norte titulado "Mini-reporteros científicos en acción" que tuvo una duración de dos semanas. El programa DeLTA parte de la premisa de que el talento académico se manifiesta por una "habilidad académica significativamente superior que posee una persona en relación a sus pares, en este caso los niños y niñas seleccionados (López, Arancibia y Bralic, 2002; Flanagan y Arancibia, 2005). En este caso, los niños y niñas que han participado de la experiencia tienen un dominio destacado de conocimientos, destrezas o habilidades y han elegido entre diversas ofertas formativas el curso de periodismo científico.

\section{Intervención}

La intervención se ejecutó, específicamente, en un curso del curso "Mini-reporteros científicos en acción" ha sido diseñado para potenciar el talento académico de los y las estudiantes, ya que su desarrollo solo es posible "cuando el individuo interactúa de manera activa con el ambiente y está abierto a recibir la estimulación" (Blumen, 2008:153). Este curso en su versión 2014 consistió en diez jornadas completas realizadas durante el mes de enero del año 2014. El curso, tuvo como objetivo que los alumnos y alumnas conocieran los roles sociales del periodismo científico y las técnicas básicas de la comunicación social. El programa del curso estuvo conformado por 5 unidades principales que se definieron de la siguiente manera: Introducción al periodismo científico, Desarrollo de notas informativas, Entrevista a científicos regionales, Visitando la televisión y la radio y, finalmente, Edición del Boletín Verde.

El primer día de clases se aplicó el primer DAST en el aula y luego de ello, los estudiantes conocieron las diferencias entre el periodismo y divulgación de la ciencia, a través de material audiovisual e experiencial. A lo largo del curso aprendieron las herramientas básicas para redactar una nota informativa. Asimismo, adquirieron nociones fundamentales para desarrollar entrevistas a científicos, junto con definir roles para el 
quehacer periodístico. Es decir se dividieron las tareas periodísticas entre editores, reporteros, gráficos y diseñadores. También visitaron dos medios de comunicación de la región: la radio de la Universidad Católica del Norte y Antofagasta Televisión. Fue allí donde conversaron con periodistas, hicieron pruebas de cámara y lecturas en radio, lo que permitió conociendo la comunicación científica desde la práctica periodística.

Las entrevistas a científicos fueron una parte fundamental de la intervención en la mitad del programa. En esta unidad los y las estudiantes entrevistaron a un investigador del área astronómica, a una químico y a una acuicultora, cuyas edades fluctuaban entre los 30 y 45 años y además, desempeñaban labores científicas en la región donde se aplicó la herramienta. Cada entrevista grupal tuvo una duración aproximada de 60 minutos y en ellas los/as científicos participantes se mostraron accesibles y dispuestos a responder las preguntas de las y los estudiantes, generándose una conversación fluida entre los dos actores involucrados. Cada encuentro se realizó en la sala de clases y dentro de las preguntas más recurrentes que las y los estudiantes realizaron, se encontraban las de que aducían a la trayectoria de vida de los y las profesionales: ¿Por qué decidiste dedicarte a las ciencias?, ¿Te gustaban las ciencias cuando niño?, ¿Qué haces en tu trabajo?, ¿Cuántos hijos tienes?, ¿Tienes animales? 0 ¿Te gusta el deporte?. Tras todo el trabajo realizado en el curso, las y los estudiantes diseñaron un boletín que contenía las entrevistas realizadas los tres científicos regionales y las experiencias vivenciadas en las salidas a terreno. Fue en el día diez, último día del curso, en el que se realizó la segunda y última evaluación DAST.

La intervención se centró en dos aspectos claves que se diferencian de estudios precedentes enfocados únicamente en "charlas expositivas de científicos o científicas". Estos dos aspectos claves son 1) el desarrollo de capacidades comunicacionales entre los/as participantes, desde el enfoque del periodismo social y crítico y 2) diálogos participativos de personas de distintos géneros que realizan actividades científicas como trabajo principal residentes de la misma región que los/as participantes. En estos diálogos participativos los/as estudiantes pudieron preguntar aspectos amplios y cotidianos sobre la práctica científica y la vida personal de las personas que realizan actividades científicas, conociendo el quehacer científico integrado a la vida de quienes lo realizan. Los y las estudiantes no fueron meros espectadores pasivos, sino comunicadores empoderados en un rol de censores y constructores de opinión pública. A partir de esto, entrevistaron a tres profesionales entre los 30 y 45 años de la Región de Antofagasta: un astrónomo, una química y una acuicultora. En las tres entrevistas, las y los estudiantes desempeñaron roles activos como periodistas e realizaron preguntas y contra preguntas, tomando el control de este proceso, buscando en estas fuentes explicaciones sobre las prácticas científicas.

Es importante advertir que, si bien las representaciones sociales son difíciles de transformar, en tanto no es un proceso inmediato y radical y eventualmente se pueden producir brechas entre discursos y prácticas, este tipo de intervenciones permite recordar que éstas en sí, son fenómenos dinámicos, sociohistóricamente construidos y que poseen la capacidad de transformación o por lo menos de problematización de lo presupuesto o aquello que se da por sentando (Marcová, 2006). De esta manera, es posible co-construir nuevas formas de saber social, toda vez que se dan ciertas condiciones observadas durante el proceso, como las que se señalan a continuación:

\section{a) Problematización de situaciones hegemónicas}

El proceso de intervención realizado se planteó desde un comienzo como una instancia que permitiera reflexionar críticamente con sus participantes las lógicas adultocéntricas, que sitúan a los/as niños/as como entes pasivos respecto a ciertas disciplinas y saberes. Así desde una perspectiva de derechos basada en la participación infantil se dio inicio al taller, el cual permanentemente, incentivó la problematización de normas hegemónicas asociadas a las ciencias y las relaciones de género, considerando que el rol de la mujer científica ha sido invisibilizado, teniendo mayor presencia los hombres en la información científica, siendo los medios de comunicación un actor fundamental en la valoración de este quehacer (Aladro et.al, 2014). En este sentido, se propició el cambio de normativas grupales a través del conocimiento de otras materias, en este caso, mostrando la diversidad de áreas de investigación, locaciones, prácticas y agentes, asociadas al quehacer científico y sus practicantes, promoviendo una actitud dinámica respecto de las ciencias en la actualidad.

\section{Integración de nuevos elementos y/o situaciones}

En sintonía con el primer elemento, basado en la problematización de situaciones hegemónicas, se fue coconstruyendo un escenario "adecuado" para el cambio, en que el grupo de participantes no sólo conocieran y reflexionaran respecto a nueva información, sino que desde su vivencia experimentaran la posibilidad de ejercer un rol activo y bajo un proceso de "empoderamiento" mediante talleres de comunicación, reconocieran en sí mismos/as su capacidad de agencia, la mayor parte del tiempo negada por el mundo adulto. Durante este proceso, se utilizaron ejercicios prácticos para que asimilaran las herramientas 
comunicacionales y, así, promover sus intereses por el mundo de la ciencia, entendiendo la importancia de la didactica para la apropiación de conocimientos y motivaciones por el área científica (Angarita, Fernández y Duarte, 2011). Fue así como los/as niños/as prepararon y el encuentro y contacto con las/os científicos/as invitados/as, también activando en ese momento procesos de transformación respecto a quien hace ciencia, pues ellos y ellas ya estaban experimentando otro rol en el ámbito científico, no identificado inicialmente en sus dibujos de diagnóstico.

\section{b) Contacto real y afectación emocional con el (los) fenómeno (s) a transformar}

Una vez que se han conocido y afianzado nuevos saberes, que se ha experimentado mental y corporalmente que es posible abordar la ciencia desde otras perspectivas, el contacto real y activo con otros y otras que ejercen ciencia fue clave. El contacto e interrelación posibilitó que los estereotipos sobre las prácticas disminuyeran notoriamente, puesto que la interacción cara a cara con científicos/as puede generar un cambio en la visión estereotipada que los niños/as tienen de los profesionales que desarollan la ciencia producto de las tradiciones familiares, revisión literaria o información que entregan los medios de comunicación entre otros (Medina, Middleton \& Orihuela, 2011). En el momento de preguntas a los/as invitados/as, los/as niños/as participantes del taller, desempeñaron un rol importante, problematizando de manera dialógica la labor científica y las trayectorias vitales de los/as científicos/as. Se tensionaron los niveles de prestigio de distintas disciplinas y la posición de género que les ha tocado vivir.

\section{RESULTADOS Y DISCUSIÓN}

Las primeras pruebas DAST antes de comenzar el curso, dieron cuenta de estereotipos similares a aquellos obtenidos por otras investigaciones internacionales (Flick, 1990; Finson et al. 1995; Ruiz-Mallén y Escalas 2012). Los cambios en los estereotipos y representaciones infantiles de las prácticas científicas fueron modificados, notoriamente, a lo largo de la intervención.

\section{Cambios en la dimensión de los Agentes}

Los dibujos reflejaron inicialmente representaciones sociales basadas en imágenes estereotípicas relacionadas con un hombre de mediana edad, que usa bata blanca y anteojos, cuya acción principal es la experimentación química en un laboratorio con instrumentos como probetas o tubos de ensayo, como se muestra en la Figura 1. Sin embargo, luego de las clases teóricas y prácticas de periodismo estos estereotipos disminuyeron. Esto se debió a un mayor empoderamiento de los niños y las niñas con respecto a su nuevo rol de reporteros, en tanto censores y constructores de opinión pública y un diálogo directo, activo y crítico con profesionales de la ciencia, en los cuales los y las estudiantes tenían el control de la situación.

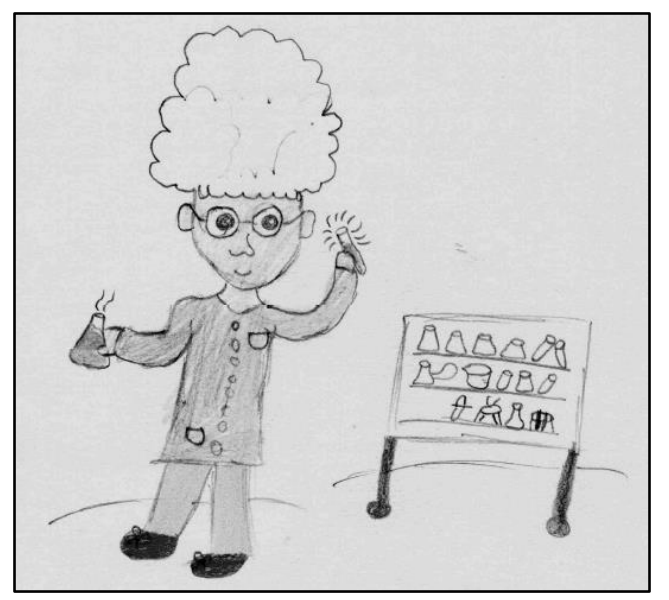

Fig. 1: imagen estereotipada de una persona que hace ciencia antes de tomar contacto directo con profesionales.

Sobre la categoría del Sexo de quiénes hacen ciencia los dibujos no muestran variación manteniendo una relación de una mujer cada cuatro hombres dibujados. Esto se condice con estudios previos que han demostrado que los estereotipos de género son, probablemente, los más difíciles de modificar (Durkin, 1985; Frueh y McGhee, 1975; Flick, 1990). Esto plantea una problemática no menor, puesto que estudios precedentes ya han constatado que una alta presencia del estereotipo de científicos varones que usan bata blanca y trabajan en el laboratorio, produce que niños y niñas piensen que sólo los hombres están destinados a ser científicos y que hacer ciencia significa estar en un laboratorio, dejando afuera un amplio abanico de prácticas, posibilidades y disciplinas que construyen el quehacer científico (Chernobilsky, 2006), como por ejemplo las ciencias sociales. 
En cuanto a la categoría de la Personalidad, la mayoría de los agentes de ciencia fueron dibujados como amigables, aunque se encontró algunos dibujos que mostraban el estereotipo de un científico alocado. La categoría de la Apariencia Personal, los científicos varones fueron dibujados con aspecto más descuidado que las mujeres. Si bien prácticamente no varió el uso de anteojos, sí hubo una significativa disminución del uso de la bata blanca.

\section{Cambios en la dimensión de los Prácticas}

En esta dimensión fue posible observar mayores cambios tras la intervención, ampliando y problematizando la percepción de la ciencia y la tecnología en su contexto social y cultural. Con respecto al área de investigación, si bien casi la totalidad de los primeros dibujos asociaban la práctica científica con las áreas de la química -mostrando experimentos en un laboratorio- tras el contacto con los científicos y científicas de la zona geográfica analizada, este estereotipo de la ciencia como actividad predominantemente de experimentación química, disminuyó más de la mitad. Todo ello permitió abrir representaciones y categorías emergentes para la investigación en el campo de la ingeniería, geología, física, astronomía y la investigación multidisciplinar. Las Figuras 2 y 3 - presentadas a modo de ejemplo- son dibujos realizados por el mismo participante, al comienzo y al final del curso, respectivamente, y muestran los cambios en las representaciones a nivel de prácticas científicas.



Fig. 2: Cambios a nivel de prácticas científicas



Fig. 3: Cambios a nivel de prácticas científicas. 
En la Fig. 2 es posible ver a un científico en el laboratorio con herramientas del área química y luego, en la Fig. 3, se visualiza a un científico con vestimenta más casual, sin bata y con herramientas de diferentes disciplinas (un telescopio con el cual observa los astros como el sol y algunos planetas) y herramientas de laboratorio químico. También se representa un cambio en cuenta a la actividad realizada, pasando de la experimentación (Fig. 2) a la inclusión de la observación como actividad científica (Fig. 3). Esto permite interpretar que tras la intervención es posible una ampliación en la percepción de las áreas, actividades y herramientas propias de la investigación de los profesionales de la ciencia. La diversidad de disciplinas con las que tuvieron contacto los y las estudiantes, la posibilidad de abrirse a otras ciencias y a otros modos de hacer ciencia, más allá de sus ideas iniciales, produjo una disminución notoria en cuanto a las categorías referidas a locaciones y herramientas científicas, pues el laboratorio, que fue la locación preferida en la mayoría en los dibujos iniciales, disminuyó.

Como ejemplifican las Figuras 4 y 5, tras la intervención los niños y niñas comenzaron a considerar como locación del trabajo científico también las oficinas y salas de clases (Fig. 5), que se condice con la institucionalidad científica chilena que se desarrolla en un $70,4 \%$ dentro de las universidades y que en gran medida supedita la investigación científica a la práctica académica-docente (Sebastián, 2014). Los instrumentos de laboratorio químico como instrumental científico prototípico, relacionado con tubos de ensayo y probetas (Fig. 2), se redujeron a la mitad tras la intervención. La intervención centrada en el contacto personal con científicos y científicas, que explicaban sus prácticas y quehaceres diarios, les permitió a los y las estudiantes conocer otro tipo de herramientas e instrumentos usados por científicos y científicas, tales como computadores, máquinas, calculadoras, microscopios, telescopios, libros, manuscritos, pizarras, lupas, etc. De igual manera, al final del curso, se encontraron dibujos sobre quehacer científico que incluían las áreas de la ingeniería, energías renovables, matemáticas, biología, geología, astronomía, entre otras.

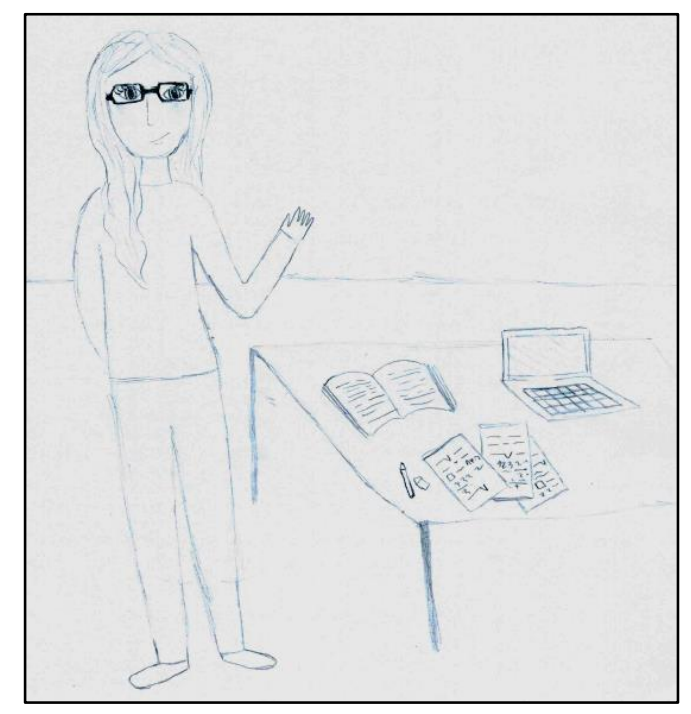

Fig. 4: cambios en la categoría de Locación de trabajo, incluyendo la oficina y el aula.



Fig. 5: cambios en la categoría de Locación de trabajo, incluyendo la oficina y el aula. 
De esta forma, es posible constatar que la intervención permitió que niños y niñas se vincularan con aquello que estaban experimentando, no solo intelectualmente sino afectivamente, propiciando así la transformación de representaciones sociales respecto a la ciencia. En los primeros dibujos, las personas que hacen ciencia fueron representadas distantes y distintivas con batas blancas y alejadas del mundo a través de laboratorios asépticos. En sus dibujos post-intervención, los/as niños/as representaron otros escenarios y actividades científicas, cambiaron las representaciones de género e incluso sus vestimentas eran más cercanas a las que ellos/as mismos utilizan en la vida cotidiana, subvirtiendo así la distancias y asepsia científica instalada inicialmente.

\section{CONCLUSIONES}

De acuerdo a las representaciones sociales sobre la labor científica y los roles que se desempeñan dentro de ésta, la intervención mostró que 1) la imagen estereotipada que los niños y niñas tenían sobre la ciencia presentó cambios importantes tras la intervención efectuada en cuanto a las prácticas científicas, en la medida en que los participantes estuvieron en contacto directo y activo con científicas y científicos de su región. 2) Tras la intervención no fue posible disminuir los estereotipos de género, lo cual plantea desafíos a futuro en diseño de programas de intervención y educación científica en la población infanto juvenil a fin de estimular un mayor interés de las niñas en el área científica.

\section{REFERENCIAS}

Aladro, E., G. Padilla, P. Requeijo, D. Semova, J.García, M.T. García y M. Viñarás, La presencia y representación de la mujer científica en la prensa española, Revista Latina de Comunicación Social, 69, 176-194 (2014).

Andre, T., M. Whigham, A. Hendrickson y S. Chambers, Competency beliefs, positive affect and gender stereotypes of elementary students and their parents about science versus other school subjects, Journal of Research in Science Teaching., 36, 719-747 (1999).

Angarita-Velandia, M. A., F. H. Fernández-Morales y J. E. Duarte, Utilización de material didáctico para la enseñanza de los conceptos de ciencia y tecnología en niños, Rev. Investig. Desarro. y Innov. 2(1): 35-43 (2011)

Barman, C, Students' views about scientists and school science, Engaging K-8 teachers in a national study, Journal of Science Teacher Education, 10, $43-54$ (1999).

BERA-British Educational Research Asociation, Ethical Guidelines for Educational Research, BERA, Londres (2011).

Bravo Gónzalez, P., C. Gónzalez Weil, V. López Leiva y M. Martínez Larrain, ¿Qué Está Haciendo El Científico: Análisis de La Actividad Científica Descrita Por Alumnos Secundarios Chilenos de $11^{\circ}$ Y $12^{\circ}$ Grado de Distintos Tipos de Establecimientos Educacionales, VIII Congreso Internacional sobre Investigación en la Didáctica de las Ciencias: 2948-2952 (2014)

Buldu, M, Young children's perceptions of scientists, A preliminary study. Educational Research, 48(1), 121132 (2006).

Calabrese Barton, A, Science education in urban settings, seeking new ways of praxis through critical ethnography, Journal of Research in Science Teaching, 38, 899-917 (2011).

Chambers, D, Stereotypic images of the scientist, The Draw-a-Scientist Test. Science Education, 6, 255-265 (1983).

Chavous, T, African American college students in predominantly White institutions of higher education. Considerations of race and gender, Perspectives, 8, 142-150 (2002).

Chavous, T., D. Rivas-Drake, C. Smalls, T. Griffin y C. Cogburn, Gender matters, too, the influences of school racial discrimination and racial identity on academic engagement outcomes among African American adolescents, Developmental Psychology, 44, 637-654 (2008).

Chernobilsky, L.B., El uso de la computadora como auxiliar en el análisis de datos cualitativos, in Estrategias de investigación cualitativa by I. Vasilachis de Gialdino (coord.), pp. 239-262, Gedisa, Barcelona (2006).

Christidou, V, Greek students' science-related interests and experiences, Gender differences and correlations, International Journal of Science Education, 28, 1181-1199 (2006). 
Christidou, V., V. Hatzinikita, y G. Samaras, The image of scientific researchers and their activity in Greek adolescents' drawings. Public Understanding of Science, 21(5), 626-647 (2012).

Christidou, V, Interest, attitudes and images related to science. Combining students' voices with the voices of school Science, teachers, and popular science, Int. J. Environ. y Science Education, 6(2), 141-159 (2011).

Cortassa, C., El aporte de la Teoría de las Representaciones Sociales a los estudios de Comprensión Pública de la Ciencia, Revista de Ciencia, Docencia y Tecnología, 21 (40), 9-44, (2010).

Domínguez-Gutiérrez, S. Las representaciones sociales en los procesos de comunicación de la ciencia, I Congreso iberoamericano de Ciencia, Tecnología, Sociedad e Innovación; (OEI), D.F., México (2006).

Finson, K, Applicability of the DAST-C to the images of scientists drawn by students of different racial groups, Journal of Elementary Science Education, 15, 15-26 (2001).

Finson, K, Drawing a scientist, What we do and do not know after fifty years of drawings, School Science and Mathematics, 102, 335-345 (2002).

Finson, K., J. Beaver y B. Cramond, Development and field-test of a checklist for the Draw-a-Scientist-Test, School Science y Mathematics, 95(4), 195-205 (1995).

Flick, L, Scientist in residence program improving children's image of science and scientists, School Science and Mathematics, 90, 204-214 (1990).

Fort, D.C. y H.L. Varney, How students see scientists, Mostly male, mostly white, and mostly benevolent, Science and Children, 26, 8-13 (1989).

Fung, Y, A comparative study of Primary and Secondary School Students' Images of Scientists, Research in Science and Technological Education, 20, 199-213 (2002).

Jodelet, D, Aportes del enfoque de las representaciones sociales al campo de la educación, Espacios en Blanco, 21, 133-154 (2011).

Joyce, B. y S. Farenga, Informal science experience, attitudes, future interest in science, and gender of highability students, an exploratory study, School Science and Mathematics, 99, 431-437 (1999).

Lay, S.L. y M. Montañes, Las representaciones sociales del mundo adulto sobre la infancia y la participación infantil, Salud y Sociedad, 4(3), 304-316 (2013).

Manzini, S. Effective communication of Science in a culturally diverse society. Science Communication, 25, 191-197 (2003).

Manzoli, F., Castelfranchi, Y., Gouthier, D. \& Cannata, I, Children's perceptions of science and scientists: A case study based on drawings and story-telling, 9th International Conference on Public Communication of Science and Technology, Seúl (2006).

Marková, I, Dialogicidade e representações sociais, as dinâmicas da mente, Vozes, Brasil (2006).

Mason, C.L., J.B. Kahle, y A.L Gardner, Draw-A-Scientist Test, Future implications. School Science and Mathematics, 91, 193-198 (1991).

Mattern, N. y C. Schau, Gender differences in science attitude-achievement relationships over time among white middle-school students, Journal of Research in Science Teaching, 39(4), 324-340 (2002).

Mayorga Rojel, A. y C. León, El malvado siempre es el otro, Perú y su construcción discursiva en la prensa chilena, Universum, 22(2), 164-176 (2007).

Mead, M. y R. Metraux, The image of the scientist among high school students. A pilotstudy Science, 126, 384-390 (1957).

Medina-Jerez, W., K.V. Middleton y W. Orihuela-Rabaza, Using the DAST-C to explore Colombian and Bolivian students' images of Scientists, International Journal of Science and Mathematics Education 9(3): 657-690 (2011).

Morgan, C., J.D. Isaac y C. Sansone, C, The role of interest in understanding the career choices of female and male college students, Sex Roles, 44, 295-320 (2001).

Moscovici, S. Memória, imaginário e representações sociais, Museu da República, Brasil (2005). 
Neathery, M.F, Elementary and secondary students' perceptions toward science: correlations with gender, ethnicity, ability, grade, and science achievement, Electronic Journal of Science Education, 2, 1 (1997).

Newton, D. y L. Newton, Young children's perceptions of science and the scientist, International Journal of Science Education, 14(3), 331-348 (1992).

Oaklander V. El tesoro escondido. La vida interior de niños y adolescentes, Editorial Cuatro Vientos, Chile (2008).

Oliveira, A.M, Abordagem societal das representações sociais, Sociedade e Estado, 24(3), 713-737 (2009).

Pizzo, M.E., L.N. Slobinsky y M.G. Panzera, Construcción de identidades sociales en la niñez, Diferencias educativas y pobreza. Anuario de investigaciones, 14, 185-192 (2006).

Polino, C., Los estudiantes y la ciencia: encuesta a jóvenes iberoamericanos, OEI, Buenos Aires (2011).

Pujalte, A.P., L. Bonan, S. Porro, y A. Adúriz-Bravo, Las imágenes inadecuadas de ciencia y de científico como foco de la naturaleza de la ciencia: estado del arte y cuestiones pendientes, Ciência \& Educação (Bauru), 20(3), 535-548 (2014).

Rodari, P. Science and scientists in the drawings of European children, Journal of Science Communication, 6(3), 1-12 (2007).

Rubin, E, V. Bar y A. Cohen, The images of scientists and science among Hebrew- and Arabic-speaking preservice teachers in Israel, International Journal of Science Education, 25(7), 821-846 (2010).

Ruiz-Mallén, I. y M.T. Escalas, Scientists Seen by Children, A Case Study in Catalonia, Spain, Science Communication, 34(4), 520-545 (2012).

Schibeci, R.A. y J.P. Riley, Influence of students' background and perceptions on science attitudes and achievement, Journal of Research in Science Teaching, 23(3), 177-187 (1986).

Schibeci, R. y I. Sorenson, I. Elementary school children's perceptions of scientists. School Science and Mathematics, 83(1), 14-19 (1983).

Sebastián, J., Apuntes sobre las trayectorias y condicionantes del desarrollo científico de Chile in Socializar Conocimientos №2, Observando a Chile desde la Distancia by L. Valderrama y B. Santander, Boris (Eds.), pp. 19-24, Redlnche Ediciones, Santiago de Chile (2014).

She, H-C, Gender and grade level differences in Taiwan students' stereotypes of science and scientists, Research in Science and Technological Education, 16(2), 125-135 (1998).

Song, J. y K. Kwang-Suk, How Korean students see scientists, the images of the scientist, International Journal of Science Education, 21(9), 957-977 (1999).

Sumrall, W, Reasons for the perceived images of scientists by race and gender of students in grades 1-7, School Science and Mathematics, 9(2), 83-90 (1995).

Torres, C., Representaciones sociales de la ciencia y la tecnología, Revista Española de Investigaciones Sociológicas, 111, 9-43, (2005).

Tsai, C., Nested epistemologies: science teachers' beliefs of teaching, learning and science, International Journal of Science Education, (24 (8), 771-783, (2002).

Van Dijk, T. Discurso y Poder, Editorial Gedisa, España (2009).

Vernal, T. y L. Valderrama, La percepción de la ciencia y de científicos/as en un país austral. El caso de DeLTA Chile, Revista Tercer Milenio, 13(28), 42-50 (2009).

Walsh, C, Interculturalidad y decolonialidad, perspectivas, críticas y políticas, Revista Visão Global, 15(1-2), 61-7 (2012).

Wagner, W., Flores-Palacios, F., Apuntes sobre la epistemología de las representaciones sociales, Revista de Educación Matemática, 22 (2), 139-162, (2010).

Weinburgh, M.H,The effects of systemic reform on urban, African American fifth grade students' attitudes toward science, Journal of Women and Minorities in Science and Engineering, 9(1), 53-72 (2003).

Weinburgh, M.H. y D. Steele, D The modified attitudes toward science inventory, Developing an instrument to be used with fifth grade urban students, J. Women and Minorities in Science y Eng., 6(1), 87-98 (2000). 
